\title{
NON-DESTRUCTIVE EVALUATION OF RESCUE AND PATROL UNMANNED AERIAL VEHICLE
}

\author{
Jowita Pawluczy-Majka \\ Rzeszow University of Technology \\ Department of Aircrafts and Aircraft Engines \\ Powstancow Warszawy Street 12, 35-959 Rzeszow, Poland \\ tel.: +48126282116 , fax: +48126282037 \\ e-mail: jowita.pawluczy@prz.edu.pl
}

\begin{abstract}
With the growing intensity of civil usage of the UAV, one of the most important problems is safety. It consists of operational safe use of the UAV in the common air area and reliability of the aerial vehicle construction.

Each flying object is subjected to external loads, which influence on it in flight and on the ground. The external loads are mainly caused by aerodynamic, weight and inertia influence, ground reaction force and the power unit [1]. That is why during service, UAV structures are prone to many mechanical and environmental conditions that can damage to composite structures in the form of delamination, fiber breakage, and matrix cracking. Monitoring the level and type of damage to a composite structure is vital to determining the component's structural integrity and preventing the failure of the structure during flight [2].

Non-destructive testing which has been used so far to identify defects in the aircraft structure made from metallic materials has a quite limited application in the case of composite structures. With the growing use of composites in the aviation industry, a necessity appears to work out a method allowing identification of technological defects and damages appeared in the process of the UAV's operation. The answer to the appearing demand could be, for instance, the active thermography technique used for the thin wall test of the skin elements of the UAV.

The obtained research results bring closer the problem of selection of the quick, well-fitted non-destructive testing method depending on the kind of the diagnosed construction.
\end{abstract}

Keywords: UAV, NDT diagnostics, active thermography

\section{Introduction}

In the conditions of the growing intensity of the UAV usage one of the most important problems is the improvement of their technical and performance effectiveness. Unmanned aerial vehicles (UAV) are one of the fastest developing branches of aviation. The drones have been built for military purposes so far. However, civil use of UAV is becoming more and more attractive regarding their unique abilities following from the lack of crew risk when performing dangerous tasks, unlimited flight lengths and so on. More than that, the progress in the range of steering systems and data transmission systems, as well as miniaturizing of the electronic equipment (e.g. sensors) caused that the UAV gained a lot of useful potential. It occurs that the UAV are more beneficial in long-term use than their manned counterparts, mainly light planes and helicopters are, although the costs of their design and purchase are still high.

Each aircraft is subjected to external loads caused by aerodynamic, ground reaction force, inertia force, power plant etc.

The UAV performing the flights over the densely populated areas must be characterized by a similar to manned aircraft safety level. As there are no specified regulations for mini UAV construction as well as regulations which determine the requirements of reliability and safety to be met the existing regulations for the manned light and very light aircraft construction are used. However, not all the requirements stated in these regulations reflect the real state of the loads to which the drone is subjected [3]. 


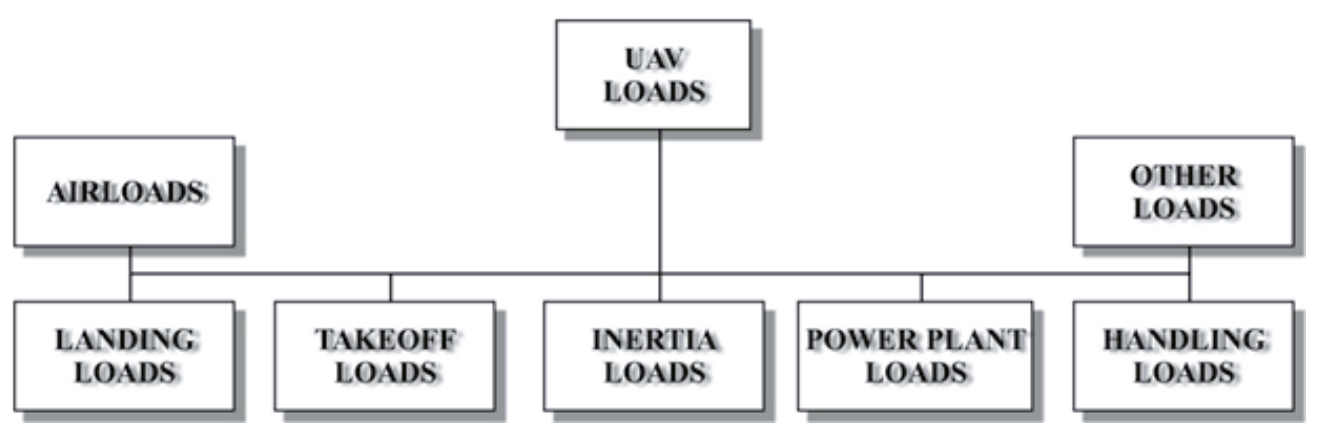

Fig. 1. UAV external loads [6]

Also, the processes of advanced composite manufacture are prone to errors. Many defects cause a reduction in the mechanical properties of a composite structure. In many cases it could lower the properties the design allowable, hence the importance of detecting defects using nondestructive technologies.

Non-destructive testing which has been used so far to identify defects in the aircraft structure made from metallic materials has a quite limited application in the case of composite structures. With the growing use of composites in the aviation industry, a necessity appears to work out a method allowing identification of technological defects and damages appeared in the process of the UAV's operation. The answer to the appearing demand could be, for instance, the active thermography technique used for the thin wall test of the skin elements of the UAV. The aim of this work was to bring closer the problem of selection of the quick, well-fitted non-destructive testing method depending on the kind of the diagnosed construction. The obtained results indicate the potential of Infrared Thermography methods for the detection of flaws in aircraft components causing critical defects in these structures. This article presents the results of research with use of pulse thermography technique to identify defects like voids, fracture and change of thickness in the sandwich composite aircraft structures.

\section{Problem identification}

Local over or underthickness can be characterized by reduced parameter $\delta$ that is the relative thickness change $e_{d}$ with respect to the reference slab thickness $e_{0}$. Parameter $e_{d}$ will be positive for bump and negative for the region with smaller thickness. In any parameters estimation problem, a very useful insight into the possibility of identifying a parameter $\delta$ is to plot the variation of its sensitivity coefficient versus the variable [4]. The contrast is a decreasing function of time. It goes to infinity for short time, showing a strong departure from delamination contrast. The sensitivity coefficient and the contrast curves have the similar features: at any time, a $10 \%$ increase of $\delta$ produces the same value of increasing of the contrast, making therefore the estimation of $\delta$ possible.

Thermal Non Destructive Testing (TNDT) is a non-invasive inspection of materials by analysing thermal flux showed on thermogram. Generally in TNDT models differential temperature signals $\Delta \mathrm{T}$ and their derivatives, such as running temperature contrast $\mathrm{C}^{\mathrm{run}}=\Delta \mathrm{T} / \mathrm{T}_{\mathrm{nd}}$ depends on time $\left(\tau, \tau_{\mathrm{h}}\right)$, heating parameters $\left(\mathrm{Q}, \tau_{\mathrm{h}}\right)$, intensity of heat exchange between a sample and the ambient $\left(\alpha^{\mathrm{F}}, \alpha^{\mathrm{R}}\right)$, sample thickness (L), sample thermal properties $\left(\lambda\right.$, a), defect size $\left(\mathrm{h}_{\mathrm{x}}, \mathrm{h}_{\mathrm{y}}\right.$, $\left.\mathrm{h}_{\mathrm{z}}=\mathrm{d}\right)$, defect depth (1) and defect thermal properties $\left(\lambda_{\mathrm{d}}, \mathrm{a}_{\mathrm{d}}\right)$. In the TNDT method, defect detectability varies significantly in time.

On a heated sample, the boundary condition is constituted by the sample external heating and its cooling with convection and radiation flux $\mathrm{Q}_{\mathrm{cv}+\mathrm{rd}}$. The corresponding phenomena are conductive flux $\mathrm{Q}_{\mathrm{cd}}$ that penetrates the sample. The heating flux $\mathrm{Q}$ is described as in paper [5]:

$$
Q=Q_{c w+r d}+Q_{c d} \text {. }
$$


The general presentation of (1) is as follows:

$$
-\lambda \frac{\partial T(z=0)}{\partial z}=Q-\alpha\left[T(z=0)-T_{a m b}\right]
$$

where $z=0$ represent front surface of the specimen, and $z=L$ rear surface (3). On the rear surface there are only two flux of heat, therefore:

$$
-\lambda \frac{\partial T(z=0)}{\partial z}=\alpha\left[T(z=L)-T_{a m b}\right]
$$

where:

$\mathrm{L}$ - sample thickness.

Most typical TNDT defects can be considered as thin air gaps where the heat conduction flux is caused by the temperature gradient $\Delta \mathrm{T}=\mathrm{T}_{1}-\mathrm{T}_{2}$ and can be evaluated by equation (4):

$$
Q=-\lambda(\partial T / \partial x) \text {. }
$$

In the most of TNDT inspected object convection can be neglected because of Grashof and Prandtl criteria and test condition where $\mathrm{T}_{1}-\mathrm{T}_{2}<100^{\circ} \mathrm{C}$, then radiation flux in thin gas-filled voids is described by the following formula:

$$
Q_{r d} \approx 4 \delta T^{3}\left(T_{1}-T_{2}\right),
$$

where:

$$
T=\frac{T_{1}+T_{2}}{2}
$$

Therefore, the ratio between the conductive and radiation flux in a thin gas-filled void is:

$$
\frac{Q_{c d}}{Q_{r d}} \approx \frac{K}{4 d \sigma T^{3}} .
$$

In general, on the boundary between layers I and $i+1$, the continuity of both heat fluxes and temperatures is described as follow:

$$
\lambda_{i} \frac{\partial T_{i}}{\partial z}=\lambda_{i+1} \frac{\partial T_{i+1}}{\partial z}, T_{i}=T_{i+1} .
$$

\section{Experimental investigations}

Thermographic investigations can be performed in two ways by use an active or passive method. The investigation of composite materials most often demands the active method. The active approach requires an external heat source to stimulate tested materials. In this experiment reflection method was used (Fig. 2). This method is mostly applied for composites.

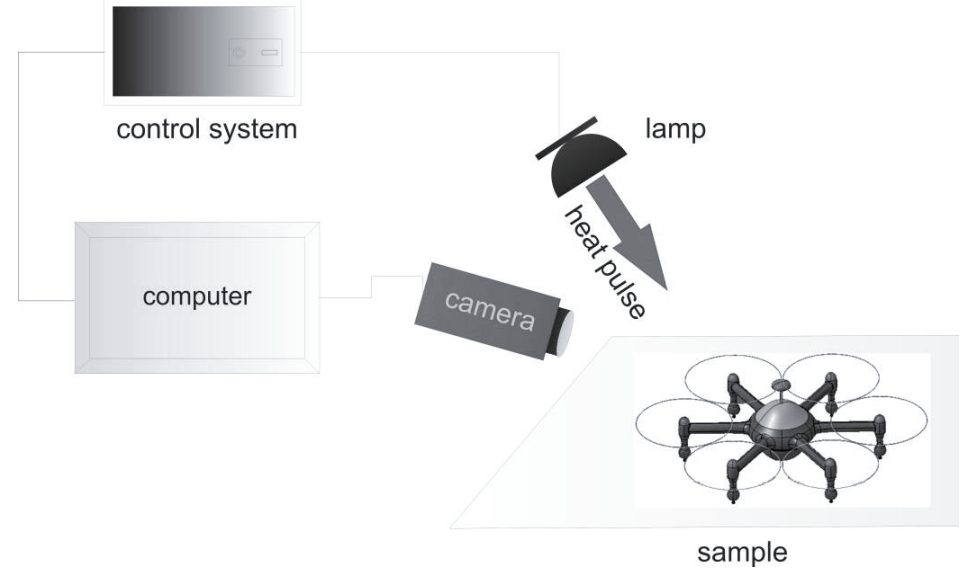

Fig. 2. Experimental setup for Reflection method in Pulse IR Thermography 
In this test a heating lamp (power $500 \mathrm{~W}$ ) was positioned $0.6 \mathrm{~m}$ before the test sample. The camera was placed $0.7 \mathrm{~m}$ from the sample. As sample (Fig. 3) was carbon-epoxy composite body of UAV Hexacopter used. Examined sample was used for normal patrol missions, and many time reach an impact loads and other damages appeared in the process of the UAV's operation.
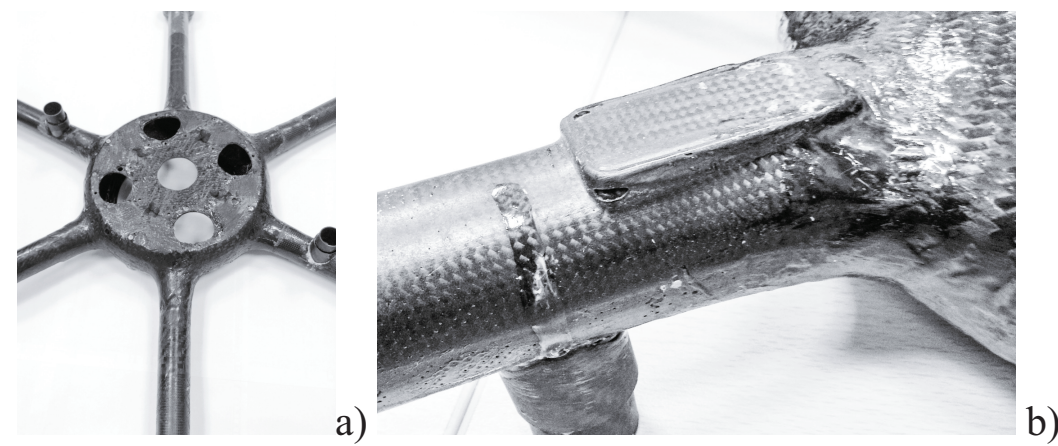

Fig. 3. Sample: carbon-epoxy UAV's body structure

Thermograms (Fig. 4 and 5) of inspected samples occurring main defects, which may cause damage of the structure. These thermograms were received during cooling phase after heating of sample and testing by the reflection method. The initial temperature of sample was $+24^{\circ} \mathrm{C}$ and the higher temperature in air-filled voids is changed to $+25^{\circ} \mathrm{C}$. Heating time was changed, because of kind of structure, between $1 \mathrm{~s}$ and $3 \mathrm{~s}$. Both phases heating and cooling were registered, but for such kind of composite structures the main important is cooling phase, where most of defects become visible.

a)
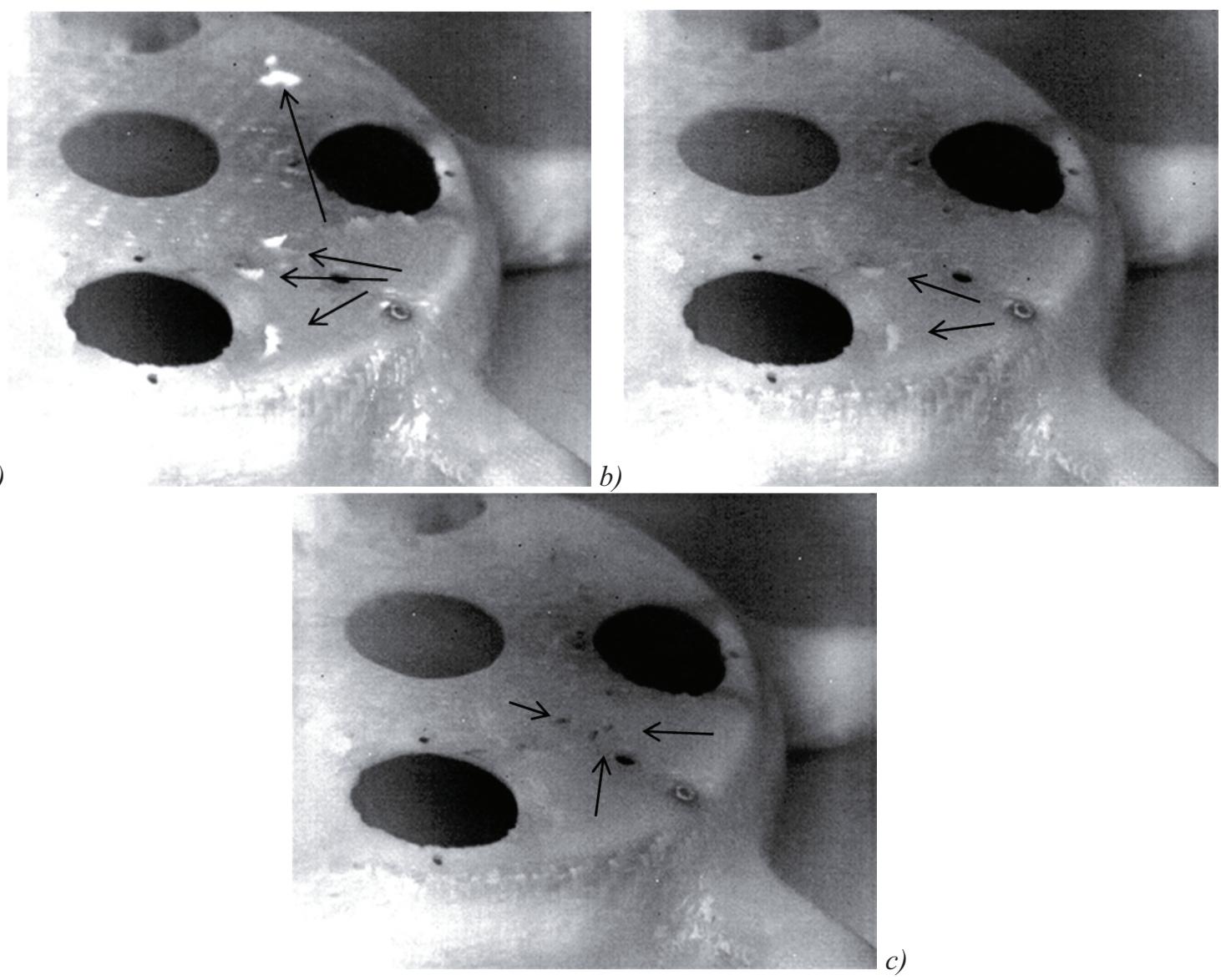

Fig. 4. Comparison of three steps of cooling phase of Pulse IR Thermography in tested carbon-epoxy sample of UAV body structure: a) air-filled voids right after heat impulse b) air-filled voids with a largest heat capacity c) crack of the structure 


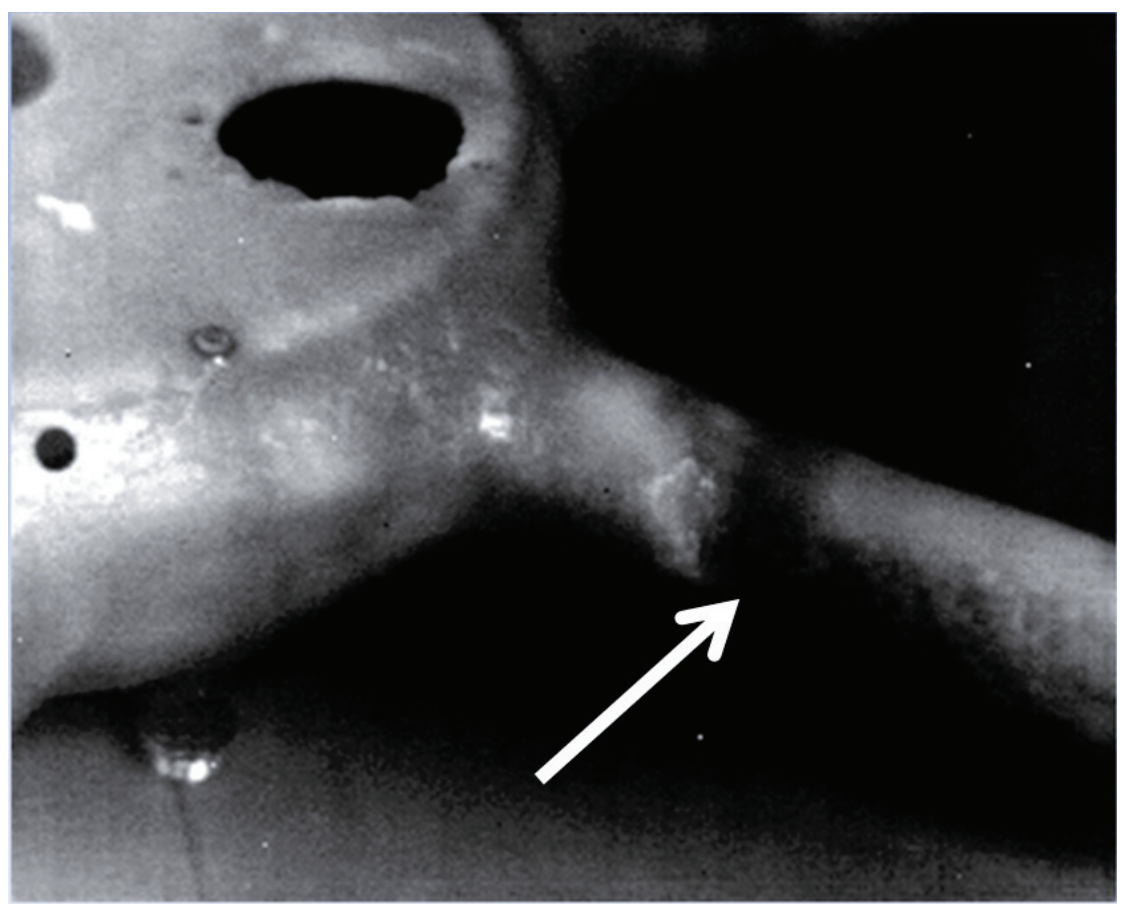

Fig. 5. Underthicknes of the carbon-epoxy structure

\section{Summary}

In this paper selected results of IR non-destructive testing the carbon-epoxy UAV's composite structure by pulse IR Thermography method is presented. Some defects appear during the process of aircraft use, but others are made already in the manufacturing process. Analysis of these results permits to qualify which regions of structure contain air-filled voids and under and overthickness defect, which may have an effect on the ability of a load-bearing structure to remain intact and functional upon the application of loads. In contrast to metals, remaining intact is not necessarily the same as remaining functionality by suffering degradation in their stiffness properties while still carrying significant loads. Thus the new methods of investigations are needed. Essential advantage of TNDT for composite structures in respect to other method of investigation like ultrasonic is high test speed.

Received results of the evaluations brings closer the answer of tested structure health, and could be used as a method for quick "in-field" inspections of the rescue and patrol UAV's. Therefore the optimal conditions of testing have to be defined separately for each specific kind of material.

\section{Acknowledgement}

The research project has been financed from a grant allocated by the National Centre for Research and Development in Poland for the years 2012-2014.

\section{References}

[1] Gundlach, J., Designing Unmanned Aircraft Systems: A Comprehensive Approach, AIAA, Inc., Reston Virginia 2012.

[2] Baker, A., Dutton, S., Kelly, D., Composite Materials For Aircraft Structures, AIAA, Inc., Reston 2004.

[3] Majka, A., Flight Loads of Mini UAV, Solid State Phenomena, T. 198, pp. 194-199, 2013.

[4] Bendada, A., Maillet, D., Degiovanni, A., Non destructive transient thermal evaluation of laminated composites: discrimination between delaminations thickness variations and multidelaminations. 
[5] Vavilov, V. P., Nondestructive testing Handbook Vol. 5 Thermal/ Infrared Testing, Spectra, Moscow 2009.

[6] Niu, M., Airframe structural design. Practical Design Information and Data on Aircraft Structures, Lockheed Aeronautical Systems Company, Burbank 1988. 